\title{
Error monitoring is related to processing internal affective states
}

\author{
Martin E. Maier ${ }^{1,2}$ - Cristina Scarpazza ${ }^{2,3}$ - Francesca Starita ${ }^{2,3}$ Roberto Filogamo $^{2}$. \\ Elisabetta Làdavas ${ }^{2,3}$
}

Published online: 19 August 2016

(C) Psychonomic Society, Inc. 2016

\begin{abstract}
Detecting behavioral errors is critical for optimizing performance. Here, we tested whether error monitoring is enhanced in emotional task contexts, and whether this enhancement depends on processing internal affective states. Event-related potentials were recorded in individuals with low and high levels of alexithymia - that is, individuals with difficulties identifying and describing their feelings. We administered a face word Stroop paradigm (Egner, Etkin, Gale, $\&$ Hirsch, 2008) in which the task was to classify emotional faces either with respect to their expression (happy or fearful; emotional task set) or with respect to their gender (female or male; neutral task set). The error-related negativity, a marker of rapid error monitoring, was enhanced in individuals with low alexithymia when they adopted the emotional task set. By contrast, individuals with high alexithymia did not show such an enhancement. Moreover, in the high-alexithymia group, the difference in the error-related negativities between the emotional and neutral task sets correlated negatively with difficulties identifying their own feelings, as measured by the Toronto Alexithymia Scale. These results show that errormonitoring activity is stronger in emotional task contexts and that this enhancement depends on processing internal affective states.
\end{abstract}

Martin E. Maier

martin.maier@ku.de

1 Department of Psychology, Catholic University of Eichstätt-Ingolstadt, Ostenstrasse 25, 85072 Eichstätt, Germany

2 Center for Studies and Research in Cognitive Neuroscience Cesena, Cesena, Italy

3 Department of Psychology, University of Bologna, Bologna, Italy
Keywords Cognitive control · Emotion · Event-related potentials (ERPs)

Error commission is particularly critical in decisions involving affective information. For example, wrongly identifying whether an approaching individual shows a happy or a fearful facial expression can have fatal consequences. Therefore, being able to detect one's own errors and adjusting behavior accordingly is crucial. In the light of this, it seems important to understand whether an affective context can alter the efficiency of error-monitoring processes, and how such alterations are produced.

An electrophysiological marker of error monitoring is the error negativity (Ne; Falkenstein, Hohnsbein, Hoormann, \& Blanke, 1990) or error-related negativity (ERN; Gehring, Goss, Coles, Meyer, \& Donchin, 1993), a negative deflection in response-locked event-related potentials that peaks over fronto-central brain areas within $80 \mathrm{~ms}$ after errors. The $\mathrm{Ne} /$ ERN is localized mainly in the anterior midcingulate cortex (aMCC; e.g., Debener et al., 2005) of the medial prefrontal cortex, and in the adjacent presupplementary motor area (SMA; Bonini et al., 2014). These regions are implicated in signaling the need for cognitive control following unfavorable behavioral outcomes such as errors (e.g., Ridderinkhof, Ullsperger, Crone, \& Nieuwenhuis, 2004).

Major theories attribute the Ne/ERN to mechanistic processes like the detection of violations of outcome predictions-for example, when an unexpected error occurs (e.g., Alexander \& Brown, 2011; Holroyd \& Coles, 2002), and the detection of posterror response conflict, when continued processing of the stimulus leads to activation of the correct response (e.g., Botvinick, Braver, Barch, Carter, \& Cohen, 2001; Yeung, Botvinick, \& Cohen, 2004). These processes are not explicitly linked to affective processing. However, 
errors themselves are aversive (e.g., Hajcak \& Foti, 2008; Riesel, Weinberg, Moran, \& Hajcak, 2013; but see Moser, Moran, Schroder, Donnellan, \& Yeung, 2014), and not surprisingly, the Ne/ERN is modulated by motivational and affective variables. For instance, the component is increased following the induction of negative affect (Pfabigan et al., 2013; Wiswede, Münte, Goschke, \& Rüsseler, 2009; Wiswede, Münte, \& Rüsseler, 2009), and in individuals with high negative affectivity (Hajcak, McDonald, \& Simons, 2004; Luu, Collins, \& Tucker, 2000) or anxiety (Hajcak, McDonald, \& Simons, 2003; Weinberg, Olvet, \& Hajcak, 2010; see Moser, Moran, Schroder, Donnellan \& Yeung, 2013, for a review). Furthermore, the Ne/ERN amplitude is larger, when errors entail more negative consequences (Hajcak, Moser, Yeung, \& Simons, 2005; Maier \& Steinhauser, 2013), and predicts priming of negative word evaluation by erroneous responses (Aarts, De Houwer, \& Pourtois, 2013).

Indeed, the aMCC has been implicated in emotional and motivational processes such as negative affect and pain (see Etkin, Egner, \& Kalisch, 2011, for a review). Based on its putative role in affective and cognitive control processes, it was proposed that the aMCC integrates information about affect, pain and the need for cognitive control to implement behavioral adjustments aimed at optimizing performance (Shackman et al., 2011). Specifically, the aMCC has strong anatomical and functional connections to motor centers, to cortical and subcortical regions involved in negative affect as well as to reinforcement-related midbrain structures. Thus, the aMCC would be optimally suited to integrate stronger affective reactions to behavioral errors with errormonitoring processes and, in this way, signal the need for behavioral adjustment following errors more efficiently. From this perspective, two testable predictions can be made. First, increasing activity in affective pathways by an emotional task context should lead to enhanced information flow between affective regions and the aMCC. Therefore, the sensitivity of the aMCC for affective reactions to errors should be increased. This increase should be reflected in an enhancement of the Ne/ERN indicating more efficient error detection. Secondly, because this process should critically depend on the functionality of affective pathways, the enhancement of the $\mathrm{Ne} / \mathrm{ERN}$ should be related to the ability to process internal affective states.

The ability to process internal affective states varies among individuals. Indeed, so-called alexithymia — that is, difficulties in identifying and describing one's own feelings (Herbert, Herbert, \& Pollatos, 2011; Parker, Keefer, Taylor, \& Bagby, 2008; Taylor, Bagby, \& Parker, 1991), is regarded as a stable personality trait expressed with varying degrees in the general population (Kokkonen et al., 2001; Salminen, Saarijarvi, Aarela, Toikka, \& Kauhanen, 1999). High levels of alexithymia are prevalent in about $10 \%$ of the general population and increase the risk for a number of psychiatric disorders (Taylor, Bagby, \& Parker, 1997). Interestingly, when highly alexithymic individuals are probed with emotional stimuli, their difficulties in identifying and describing their feelings are associated with reduced activity in brain regions including the aMCC and the dorsolateral prefrontal cortex (dLPFC; e.g., Kano et al., 2003; Karlsson, Näätänen, \& Stenman, 2008; Moriguchi et al., 2007). These are precisely the same brain regions that are also implicated in error monitoring and posterror behavioral adjustments (e.g., Kerns et al., 2004). Furthermore, alexithymia was recently shown to correlate negatively with measures of cognitive control such as conflict adaptation - that is, the reduction of interference by incongruent distractors following trials involving response conflict (De Galan, Sellaro, Colzato, \& Hommel, 2014). Thus, alexithymia is associated with reduced recruitment of brain areas associated with error monitoring and adaptive posterror adjustments and with deficits in implementing behavioral adjustments in tasks requiring cognitive control. This raises the question whether alexithymia is also associated with a deficit in error monitoring. Specifically, given the reduced capacity in alexithymia to process internal affective states, alexithymia could be associated with a reduced ability to use information about reactions of the affective system to behavioral errors for error monitoring.

The present study had two main objectives. First, we wanted to show that an emotional task context leads to increased error-monitoring activity relative to a neutral task context. This would be in accordance with the idea that a stronger activity in affective pathways boosts error-monitoring processes. Second, we aimed to test whether increased errormonitoring activity in emotional task contexts depends on the ability to process internal affective states.

To these ends, we compared the Ne/ERN of individuals with low and high levels of alexithymia (Taylor, Bagby, \& Parker, 2003) in an emotional and a neutral task context. Specifically, we administered a face word Stroop paradigm (Egner et al., 2008) involving an emotional and a neutral task set. The former required participants to classify emotional faces with respect to their emotional expression (happy or fearful), the latter with respect to their gender (female or male). Congruent or incongruent words were written across the faces to produce errors. Individuals with low levels of alexithymia were hypothesized to show larger Ne/ERN amplitudes when adopting the emotional task set than when adopting the neutral task set. This is because adopting an emotional task set should lead to stronger activation of affective pathways, and this was previously shown to enhance error monitoring. Furthermore, if processing internal affective states plays a crucial role in this process, individuals with high levels of alexithymia should not show larger Ne/ERN amplitudes with the emotional task set. Indeed, if alexithymia eliminated the impact of emotions on the Ne/ERN, this would 
suggest that the ability of performance monitoring to use affective reactions to signal errors relies on processing internal affective states.

A further event-related potential (ERP) index of error monitoring is the so-called error positivity (Pe), a parietal positivity in the response-locked event-related potential on error trials, which follows the Ne/ERN at a latency between 200 and $400 \mathrm{~ms}$ after the error. Since the Pe consistently shows larger amplitudes for errors that the participants become aware of than for errors that go unnoticed, it is commonly believed to be an index for conscious error awareness (e.g., Overbeek, Nieuwenhuis, \& Ridderinkhof, 2005; Steinhauser \& Yeung, 2010). Interestingly, early accounts of the Pe also proposed a role of this component in affective processing of errors (see Overbeek et al., 2005, for a review). Thus, we examined the $\mathrm{Pe}$ in addition to the Ne/ERN. If processing internal affective states were important for the process underlying the Pe, we would expect similar effects of alexithymia level on the Pe as on the $\mathrm{Ne} / \mathrm{ERN}$.

Finally, we examined the N450 as a correlate of conflict in the Stroop task (West \& Alain, 2000). Because the N 450 is thought to reflect conflict detection in the Stroop task (Larson, Clayson, \& Clawson, 2014), it should be suited to investigate whether alexithymia level, affective content of the task set or both are related to conflict detection in the Stroop task.

\section{Materials and method}

\section{Participants}

A total of 300 university students completed the 20 -item Toronto Alexithymia Scale (TAS-20; Taylor et al., 2003); see Table 1 for descriptive statistics. Individuals with high and low total TAS-20 scores (top quartile score $>60$; bottom quartile score $<39$ ) were selected and assigned to a lowalexithymia group (LA group) and a high-alexithymia group (HA group), respectively (e.g., Scarpazza, Di Pellegrino, \& Làdavas, 2014; Scarpazza, Làdavas, \& Di Pellegrino, 2015). The TAS-20 internal consistency, as measured by Cronbach's alpha, was acceptable (TAS total, .92; Difficulties to Identify
Feelings, .93; Difficulties to Describe One's Own Feelings to Others, .81; Externally Oriented Thinking, .65). The alexithymia module of the structured interview for the Diagnostic Criteria for Psychosomatic Research (Mangelli, Semprini, Sirri, Fava, \& Sonino, 2006), was used to confirm group membership (Grandi, Sirri, Wise, Tossani, \& Fava, 2011; Scarpazza et al., 2014). Moreover, due to the high association between alexithymia and depression (Hintikka, Honkalampi, Lehtonen, \& Viinama, 2001; Honkolampi, Hintikka, Tanskanen, Lehtonen, \& Viinamaki, 2000), the mood disorders subscale of the Structured Clinical Interview for DSM-IV Axis I Disorders (SCID-I; First, Spitzer, Gibbon, \& William, 1997/2007) was used to exclude participants with high levels of depression. Participants were included if they had no history of neurological, major medical or psychiatric disorders. In all, 20 participants were included in the LA group (mean age $=24.4$ years, $S E=1.32$ years, 16 female) and 20 participants were included in the HA group (mean age = 22.3 years, $S E=0.778$ years, 16 female). These sample sizes were determined in accordance with proposed guidelines (Simmons, Nelson, \& Simonsohn, 2011). Power analyses indicated that a sample size of 20 participants per group is appropriate for testing the main hypothesis of the present design (within-between interaction in a $2 \times 2$ repeated measures analysis of variance [ANOVA]), when medium effect sizes and power are expected. Mean age was not significantly different between groups, $p=.190$. All participants were students at the University of Bologna.

\section{Apparatus}

A PC running the Presentation software (Neurobehavioral Systems, Albany, CA) controlled stimulus presentation and response registration. Stimuli were presented on a 17 -in. color monitor.

\section{Stimuli}

Grayscale photographs of ten male and ten female faces in front view with happy and fearful expressions were taken from the Karolinska Directed Emotional Faces picture library (Lundqvist, Flykt, \& Öhman, 1998). Each face was combined

Table 1 Participants: Mean age and mean scores on the Toronto Alexithymia Scale in individuals with low and high alexithymia (LA, and HA, respectively)

\begin{tabular}{llllll}
\hline Group & Age (Years) & Total TAS-20 & DDF & DIF & EOT \\
\hline LA & $24.4(1.32)$ & $33.3(1.00)$ & $8.25(0.422)$ & $12.1(0.640)$ & $12.7(0.572)$ \\
HA & $22.3(0.778)$ & $65.4(1.05)$ & $19.2(0.659)$ & $23.8(0.794)$ & $22.5(0.790)$ \\
\hline
\end{tabular}

Total TAS-20, total score calculated by adding up the subscale scores; DDF, difficulties in describing one's own feelings to others subscale; DIF, difficulties in identifying one's own feelings subscale; EOT, external-oriented thinking subscale. Standard errors of the means are given in parentheses. See the text for further details. 
with each of the Italian words UOMO ("man"), DONNA ("woman"), GIOIA ("happiness"), and PAURA ("fear"), overlaid over the face in bold red letters. The word could either correspond with the expression or the gender of the face (congruent stimuli) or not correspond (incongruent stimuli; see Fig. 1 for examples). This resulted in 160 different stimuli. The stimuli were resized to a visual angle of $8.23^{\circ}$ height and $9.83^{\circ}$ width and presented on a black background at a viewing distance of $80 \mathrm{~cm}$. The words subtended $1.14^{\circ}$ vertically and $5.61^{\circ}$ (DONNA), $4.81^{\circ}$ (UOMO), $4.35^{\circ}$ (GIOIA), and 5.38 (PAURA) horizontally.

\section{Procedure}

On each trial, a white fixation cross was presented in the center of the screen. After $517 \mathrm{~ms}$, the stimulus was presented for $167 \mathrm{~ms}$, followed by a black screen until the participant responded. After the response, the screen remained black for 1,216 ms until the next trial started. For the neutral task set, face stimuli overlaid with the words UOMO or DONNA were presented, and participants were instructed to classify the stimuli with respect to the gender of the faces (male, female) while ignoring the words. For the emotional task set, face stimuli overlaid with the words GIOIA or PAURA were presented, and participants were instructed to classify the stimuli with respect to the emotional expression of the faces (happy, fearful) while ignoring the words. Responses had to be given

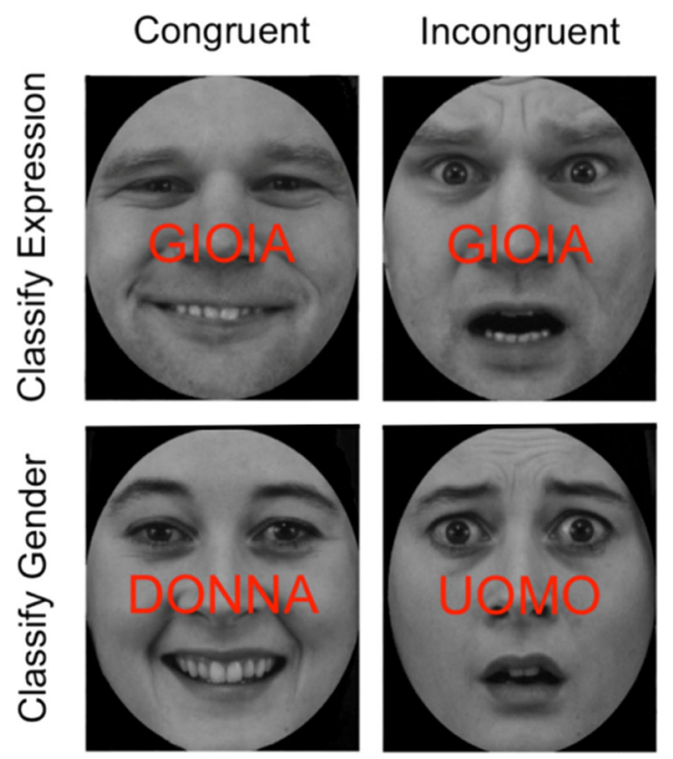

Fig. 1 Behavioral tasks. The emotional task set required classifying faces with respect to their emotional expression while ignoring the distractor words (GIOIA "happiness" or PAURA "fear"). The neutral task set required classifying faces with respect to their gender while ignoring the distractor words (UOMO "man" or DONNA "woman"). The distractor words could either correspond (congruent) or not correspond (incongruent) with the task-relevant dimension of the face. by pressing the left and right Alt keys of a standard computer keyboard with the left and right index fingers, respectively. The stimulus-response mappings were counterbalanced across participants. Each task set was maintained for eight test blocks, resulting in a total of 640 test trials per task set. Half of the participants in each group started with the emotional task set, and the other half started with the neutral task set. Each block contained 80 randomized trials (40 congruent and 40 incongruent trials). Each block began with three randomly drawn acclimatization trials, which were not considered in the data analyses. Before the test blocks, participants worked through four practice blocks of 40 trials (20 congruent, 20 incongruent) each, to practice the stimulus response mapping. Furthermore, the practice blocks were used to adjust the error rate so as to obtain a sufficient number of error trials for the analyses. To this end, participants were instructed to respond faster whenever the average error rate in a block was below $15 \%$, and to respond more accurately whenever the average error rate in a block was above $35 \%$ prior to the beginning of the next block. This speed/accuracy instruction kept the error rate between $15 \%$ and $35 \%$ and was maintained throughout the experiment. Practice blocks were excluded from the data analyses. The experiment lasted for approximately $1 \mathrm{~h} 40 \mathrm{~min}$, and small breaks were allowed between blocks if the participants so desired.

\section{Psychophysiological recording}

The electroencephalogram (EEG) was recorded with Ag/ AgCl electrodes (Fast n Easy Electrodes; Easycap, Herrsching, Germany) from 27 electrode sites (Fp1, F3, F7, FC1, FC5, C3, CP1, CP5, P3, P7, O1, AFz, Fz, FCz, Cz, Pz, Fp2, F4, F8, FC2, FC6, C4, CP2, CP6, P4, P8, O2) and from the right mastoid. The left mastoid was used as reference, and the ground electrode was placed on the right cheek. The electrooculogram (EOG) was recorded from above and below the left eye and from the outer canthi of both eyes. EEG and EOG were recorded with a band-pass filter of 0.01-100 Hz, amplified by a BrainAmp DC amplifier (Brain Products, Gilching, Germany), digitized at a sampling rate of $1000 \mathrm{~Hz}$, and resampled to $500 \mathrm{~Hz}$ offline.

\section{Data analyses}

Trials with response times (RTs) deviating more than four standard deviations from the condition mean were excluded from the RT analyses ( $<1 \%$ of all trials). Frequency data were arcsine-transformed for statistical testing (Winer, Brown, \& Michels, 1991). Mixed-model repeated measurements ANOVAs were used. Within-group planned contrasts were performed using paired-samples $t$ tests.

The EEG data were analyzed using EEGLAB, version 13.0.1 (Delorme \& Makeig, 2004), and custom routines 
written in MATLAB R2012b (The MathWorks, Natick, MA). The ERP data were re-referenced offline to the average of both mastoids, and filtered with a $0.5-$ to $30-\mathrm{Hz}$ passband. Epochs of $300 \mathrm{~ms}$ before and $600 \mathrm{~ms}$ after the response were extracted from the continuous EEG and baseline-corrected using a 300to $100-\mathrm{ms}$ preresponse window. This window was chosen to avoid cancelling out error-related activity due to a start of the $\mathrm{Ne} / \mathrm{ERN}$ before the completion of the motor response (e.g., Riesel, Weinberg, Endrass, Meyer, \& Hajcak, 2013). Epochs were excluded if the voltage on an EOG channel exceeded $400 \mu \mathrm{V}$ to remove trials with large EOG peaks. Furthermore, epochs were rejected that contained data deviating more than five standard deviations from the mean of the joint probability distribution to remove trials with improbable data. The mean percentage of excluded trials was $6.40 \%(S E=2.90 \%)$ in the LA group and $5.12 \%(S E=2.11 \%)$ in the HA group. These values did not differ significantly from each other, $p=.807$. After artifact removal, mean totals of $41.2(S E=2.59), 54.7$ $(S E=2.62), 35.7(S E=3.00)$, and $57.3(S E=3.42)$ errors remained for the analyses of error ERPs in the congruent and incongruent conditions of the emotional and the neutral task sets, respectively. The minimum number of error trials in a condition was five for one participant, but greater than 13 for all of the remaining participants. The mean numbers of errors did not differ between the LA group $(47.2, S E=2.37)$ and the HA group $(47.3, S E=2.26), F(1,38)=0.001, p=$ $.982, \eta_{\mathrm{p}}{ }^{2}<.001$. To correct remaining artifacts, the data were subjected to a temporal independent component analysis (Jutten \& Herault, 1991; Makeig, Bell, Jung, \& Sejnowski, 1996) using the infomax algorithm (Bell \& Sejnowski, 1995). The resulting component matrix was screened for independent components (ICs) representing stereotyped artifact activity, such as horizontal (saccades) and vertical (blinks) eye movements, and muscle artifacts. This was done using a multistep correlational template-matching process, implemented in CORRMAP, version 1.02 (Viola et al., 2009). Topographies of ICs labeled as artifacts by the CORRMAP procedure were visually inspected and then calculated out of the data using inverse matrix multiplication.

The $\mathrm{Ne} / \mathrm{ERN}$ was quantified as the mean voltage in a time window of -10 to $30 \mathrm{~ms}$ relative to the response, analyzed at channel FCz. The Pe was scored as the mean voltage in a time window of 200 to $400 \mathrm{~ms}$ following the response, analyzed at channel Pz. Time windows and electrodes were selected on the basis of the maximal difference between error and correct trials. Both components were analyzed on correct and error trials to examine the relative contributions of performance monitoring on error and correct trials.

Stimulus-locked epochs from -200 to $800 \mathrm{~ms}$ relative to stimulus onset were extracted from the continuous EEG, baseline-corrected using a 200-ms prestimulus window, and artifact-corrected in the same way as the response-locked data. The conflict-related N450 potential was quantified as the mean voltage in a time window of 350 to $450 \mathrm{~ms}$ on correct trials and was analyzed at channel $\mathrm{Pz}$, where the component was maximal.

\section{Results}

\section{Performance data}

Performance data are shown in Table 2. Error rates and RTs of correct responses were separately subjected to mixed-model repeated measurements ANOVAs, with the between-subjects variable group (LA, HA) and the within-subjects variables task set (emotional, neutral) and congruency (congruent, incongruent).

Error rates Error rates showed a main effect of congruency, $F(1,38)=90.5, p<.001$, denoting higher error rates on incongruent $(20.0 \%, S E=0.678 \%)$ than on congruent $(13.6 \%, S E=0.602 \%)$ trials. Furthermore, the ANOVA revealed an interaction between congruency and task set, $F(1,38)=6.36, p=.016$. The interaction denoted that the congruency effect, calculated as the difference in the error rates between incongruent and congruent trials, was larger with the neutral $(7.89 \%, S E=1.05 \%)$ than with the emotional $(4.94 \%, S E=0.846 \%)$ task set. However, the congruency effect was significant with both the neutral, $t(39)=7.93, p<.001$, and the emotional, $t(39)=$ $6.05, p<.001$, task sets. No further effects reached

Table 2 Performance data: Error rates in\% and response times in milliseconds in the low-alexithymia group (LA) and the high-alexithymia group (HA) as a function of task set (emotional, neutral) and trial type (congruent, incongruent)

\begin{tabular}{|c|c|c|c|c|c|c|c|c|}
\hline \multirow[t]{3}{*}{ Group } & \multicolumn{4}{|l|}{ Error Rate } & \multicolumn{4}{|l|}{ RT } \\
\hline & \multicolumn{2}{|c|}{ Emotional Task Set } & \multicolumn{2}{|c|}{ Neutral Task Set } & \multicolumn{2}{|c|}{ Emotional Task Set } & \multicolumn{2}{|c|}{ Neutral Task Set } \\
\hline & $\mathrm{C}$ & IC & $\mathrm{C}$ & IC & $\mathrm{C}$ & IC & $\mathrm{C}$ & IC \\
\hline LA & $13.6(1.05)$ & $18.1(1.17)$ & $11.6(1.16)$ & $20.6(1.79)$ & $423(9.42)$ & $429(11.1)$ & $434(9.12)$ & $455(11.1)$ \\
\hline HA & $15.5(1.13)$ & $20.9(1.29)$ & $13.6(1.38)$ & $20.4(1.07)$ & $422(9.32)$ & $424(10.6)$ & $411(7.36)$ & $425(9.59)$ \\
\hline
\end{tabular}

Standard errors of the means are given in parentheses. RT $=$ Response Times; $\mathrm{C}=$ Congruent; $\mathrm{IC}=$ Incongruent. 
significance, all $p s>.220$. Importantly, no main effect ( $p$ $=.220$ ) or interaction (all $p \mathrm{~s}>.256$ ) involved the variable group.

RTs of correct responses Just like the error rates, the RTs of correct responses showed a main effect of congruency, $F(1$, $38)=40.6, p<.001$, and an interaction between congruency and task set, $F(1,38)=38.1, p<.001$. The interaction denoted that the congruency effect, calculated as the difference in RTs between incongruent and congruent trials, was larger with the neutral (17 ms, $S E=2 \mathrm{~ms})$ than with the emotional (4 ms, $S E=$ $2 \mathrm{~ms}$ ) task set. However, the congruency effect was significant with both the neutral, $t(39)=7.49, p<.001$, and the emotional, $t(39)=2.19, p=.035$, task set. Furthermore, the ANOVA on RTs also revealed an interaction of task set and group, $F(1$, $38)=6.69, p=.014$. Although RTs were faster with the emotional (426 ms, $S E=7 \mathrm{~ms}$ ) than with the neutral (444 ms, $S E=$ $7 \mathrm{~ms}$ ) task set in the LA group, $t(19)=2.65, p<.016$, the RTs in the HA group were comparable between the emotional (423 ms, $S E=7)$ and the neutral $(418 \mathrm{~ms}, S E=6 \mathrm{~ms})$ task sets, $t(19)=0.889, p>.385$. The interaction between group, task set, and congruency was not significant, $F(1,28)=0.619$, $p=.436$. No further effects reached significance, all $p \mathrm{~s}>.099$.

\section{Posterror behavioral adjustments}

RTs and error rates were separately subjected to mixed-model repeated measures ANOVAs, with the between-subjects variable group (LA, HA) and the within-subjects variables task set (emotional, neutral), previous trial type (correct, error), and current congruency (congruent, incongruent).

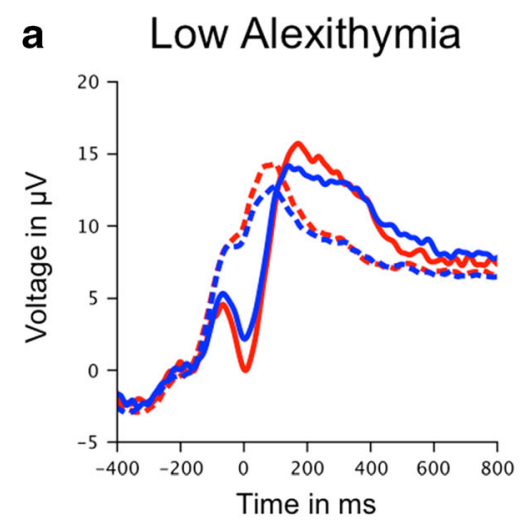

C

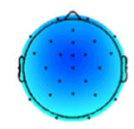

Emotional

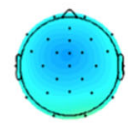

Neutral
Fig. $2 \mathrm{Ne} / \mathrm{ERN}$ data: Response-locked grand average waveforms at electrode $\mathrm{FCz}$ for correct and error trials with the emotional and with the neutral task set, for participants with low alexithymia (A) and participants with high alexithymia (B), as well as scalp topographies of

Posterror slowing RTs were slower following errors (443 ms, $S E=3.87 \mathrm{~ms})$ than following correct trials $(424 \mathrm{~ms}, S E=$ $3.55 \mathrm{~ms}), F(1,38)=32.9, p<.001, \eta_{\mathrm{p}}{ }^{2}=.464$. This posterror slowing was larger with the emotional (posterror - postcorrect $=23 \mathrm{~ms}, S E=3.62$ ) than with the neutral (posterror postcorrect $=14 \mathrm{~ms}, S E=2.28)$ task set, $F(1,38)=4.78, p$ $<.035, \eta_{\mathrm{p}}{ }^{2}=.112$. No further effects involving the variable previous trial type were significant (all $p \mathrm{~s}>.117$, all $\eta_{\mathrm{p}}{ }^{2} \mathrm{~s}<$ .063).

Posterror increase of accuracy Error rates were lower following errors $(16.5 \%, S E=0.644 \%)$ than following correct trials $(18.5 \%, S E=0.507 \%), F(1,38)=14.2, p<.001, \eta_{\mathrm{p}}{ }^{2}=$ .271 ; that is, there was a reliable posterror increase of accuracy. No further effects involving the variable previous trial type were significant (all $p \mathrm{~s}>.26$, all $\eta_{\mathrm{p}}{ }^{2} \mathrm{~s}<.037$ ).

\section{Response-locked ERP data}

Ne/ERN Clear Ne/ERNs, with more negative waveforms for errors than for correct trials starting about $50 \mathrm{~ms}$ before and lasting until $100 \mathrm{~ms}$ after the response, emerged in both groups (see Figs. 2A and B). The difference between error and correct trials was maximal at channel $\mathrm{FCz}$ (panel C). Although in the LA group the Ne/ERN seemed larger with the emotional than with the neutral task set, no such difference was evident in the HA group. A mixed-model repeated measures ANOVA with the between-subjects variable group (LA, HA) and the withinsubjects variables task set (emotional, neutral), congruency (congruent, incongruent), and response (correct, error), on the mean voltages in the Ne/ERN time window at channel

\section{b High Alexithymia}

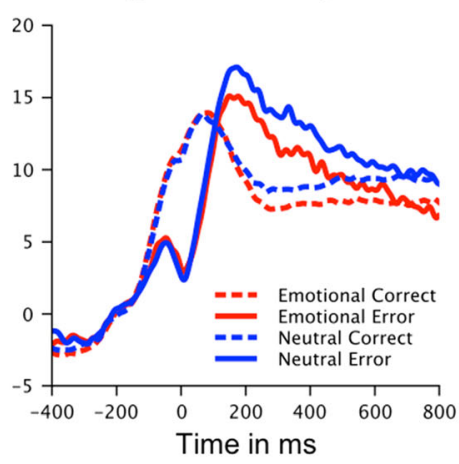

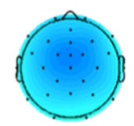

Emotional

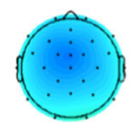

Neutral

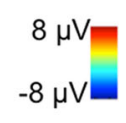

the mean differences between error and correct waveforms in a time window of -10 to $30 \mathrm{~ms}$ relative to the buttonpress $(\mathbf{C})$. Ne/ERN = error-related negativity, $0=$ time point of buttonpress, $\mu \mathrm{V}=$ microvolts 
FCz, confirmed these impressions. The ANOVA revealed a main effect of response, $F(1,38)=179, p<.001, \eta_{\mathrm{p}}{ }^{2}=.825$. Voltages were more negative on error $(2.30 \mu \mathrm{V}, S E=$ $0.463 \mu \mathrm{V})$ than on correct $(10.9 \mu \mathrm{V}, S E=0.398 \mu \mathrm{V})$ trialsthat is, a reliable Ne/ERN was observed. Furthermore, an interaction between task set and response, $F(1,38)=8.71, p=$ $.005, \eta_{\mathrm{p}}{ }^{2}=.825$, and crucially, an interaction between group, task set, and response, $F(1,38)=10.0 p=.003, \eta_{\mathrm{p}}{ }^{2}=.208$, were revealed. The interaction between group, task set, and response justified analyzing correct and error trials separately for both groups. In the LA group, the mean voltage in the time window of -10 to $30 \mathrm{~ms}$ relative to the response on correct trials did not differ significantly between the emotional and the neutral task set, $t(19)=1.67, p=.111, d=0.207$ (see Table 3). By contrast, the mean voltage in this time window on error trials was significantly smaller with the emotional than with the neutral task set, $t(19)=2.51, p=.021, d=$ 0.382 (see Table 3). Thus, the Ne/ERN on error trials was significantly larger (i.e., more negative) with the emotional than with the neutral task set. In the HA group, a different picture emerged. Neither the mean voltage on correct trials nor the mean voltage on error trials differed significantly between the emotional task set and the neutral task set, $t(19)=$ $0.644, p=.527, d=0.085$, and $t(19)=0.636, p=.533, d=$ 0.091 , respectively (see Table 3 ). Thus, in the HA group, neither correct nor error trials were significantly affected by the affective content of the task set.

Furthermore, the ANOVA also revealed an interaction between congruency and response, $F(1,38)=42.0, p=.001, \eta_{\mathrm{p}}{ }^{2}$ $=.525$. The mean voltage in the $\mathrm{Ne} / \mathrm{ERN}$ time window was more positive for congruent $(11.5 \mu \mathrm{V}, S E=0.575 \mu \mathrm{V})$ than for incongruent $(10.29 \mu \mathrm{V}, S E=0.545 \mu \mathrm{V})$ stimuli for correct trials, but more positive for incongruent $(3.12 \mu \mathrm{V}, S E=$ $0.612 \mu \mathrm{V})$ than for congruent $(1.48 \mu \mathrm{V}, S E=0.688 \mu \mathrm{V})$ stimuli on error trials. No further effects emerged, all $p s>.224$, $\eta_{\mathrm{p}}{ }^{2} \mathrm{~s}<.039$.

The analyses of the RTs of correct responses revealed that in the LA group, RTs were faster with the emotional than with the neutral task set, whereas in the HA group, RTs were

Table 3 ERP data: Mean voltage in the time window of the Ne/ERN from -10 to $30 \mathrm{~ms}$ relative to the response in the low-alexithymia group (LA) and the high-alexithymia group (HA) as a function of task set (emotional, neutral) and trial type (correct, error)

Group Mean Voltage

\begin{tabular}{llrlll}
\cline { 5 - 6 } & \multicolumn{2}{ll}{ Emotional Task Set } & & \multicolumn{2}{l}{ Neutral Task Set } \\
\cline { 2 - 3 } \cline { 5 - 6 } & Correct & \multicolumn{1}{l}{ Error } & & Correct & \multicolumn{2}{l}{ Error } \\
\hline LA & $10.8(0.789)$ & $0.449(0.893)$ & & $9.73(0.895)$ & $2.56(0.864)$ \\
HA & $11.8(0.787)$ & $3.35(0.893)$ & & $11.4(0.692)$ & $2.84(0.963)$ \\
\hline
\end{tabular}

Standard errors of the means are given in parentheses. comparable between both task sets. This could indicate that for the LA group, the task with the emotional task set was easier than the task with the neutral task set. Because it is known that increasing task difficulty can lead to decreased Ne/ERN amplitudes (Kaczkurkin, 2013; Schreiber, Endrass, Weigand, \& Kathmann, 2012), we investigated whether performance differences between the task sets could account for the effect of task set on the Ne/ERN. To this end, we conducted an analysis of covariance (ANCOVA) comprising all factors of the original ANOVA, plus the RT of correct responses as a covariate. Since differences in RTs between the emotional and neutral task sets capture potential effects of task difficulty between the task sets, this analysis partialed these effects out. The ANCOVA fully replicated the critical interaction between group, task set, and response, $F(1,37)=8.96, p=.005, \eta_{\mathrm{p}}{ }^{2}=$ .195. Analogous results were obtained when error RT $[F(1$, $\left.37)=9.47, p=.004, \eta_{\mathrm{p}}{ }^{2}=.204\right]$ and error rate $[F(1,37)=$ 9.62, $\left.p=.004, \eta_{\mathrm{p}}{ }^{2}=.206\right]$ were used as covariates in the ANCOVA. This makes it unlikely that performance differences can account for the differences found between the two groups on the $\mathrm{Ne} / \mathrm{ERN}$.

Pe In a time window of 200 to $400 \mathrm{~ms}$ following the response, the waveforms were more positive for errors than for correct responses (see Figs. 3A and $\mathrm{B}$ ). This difference - that is, the $\mathrm{Pe}$ - was maximal at channel $\mathrm{Pz}$ (panel C). A mixed-model repeated measures ANOVA with the between-subjects variable group (LA, HA) and the within-subjects variables task set (emotional, neutral), congruency (congruent, incongruent), and response at channel $\mathrm{Pz}$ revealed a main effect of response, $F(1,38)=160, p<.001, \eta_{\mathrm{p}}{ }^{2}=.808$. Voltages were more positive on error $(10.6 \mu \mathrm{V}, S E=0.468 \mu \mathrm{V})$ than on correct $(3.65 \mu \mathrm{V}, S E=0.366 \mu \mathrm{V})$ trials - that is, a clear Pe was present. However, we found no significant interaction between group and response, $p=.455, \eta_{\mathrm{p}}{ }^{2}=.015$, indicating that the Pes were comparable between the LA and HA groups.

Furthermore, the ANOVA revealed an interaction of group and task set, $F(1,38)=6.26, p=.017, \eta_{\mathrm{p}}{ }^{2}=.141$. Although the waveforms were less positive with the emotional $(6.33 \mu \mathrm{V}$, $S E=0.692 \mu \mathrm{V})$ than with the neutral $(7.88 \mu \mathrm{V}, S E=$ $0.736 \mu \mathrm{V})$ task set in the HA group, $t(19)=2.75, p=.013, d$ $=0.322$, no such effect was observable in the LA group (emotional task set, $7.37 \mu \mathrm{V}, S E=0.701 \mu \mathrm{V}$; neutral task set, $7.00 \mu \mathrm{V}, S E=0.708 \mu \mathrm{V}), p>.489, d=0.072$. None of these effects changed when correct RTs, error RTs, or error rates were inserted into ANCOVAs comprising the original factors.

\section{Correlations between response-locked ERP data and alexithymia scores}

To examine whether the Ne/ERN on error trials was associated with alexithymia on an individual level, Pearson correlations between the effect of task set on the Ne/ERN (i.e., the 


\section{a Low Alexithymia}

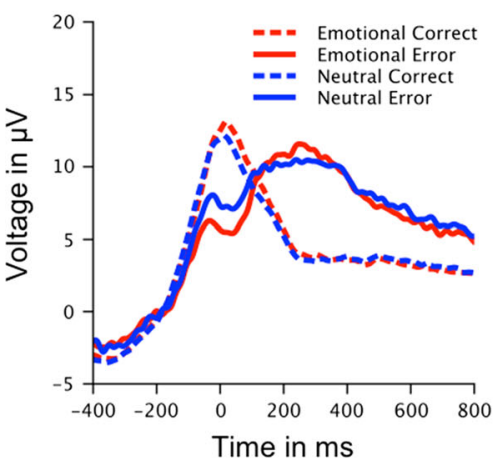

C

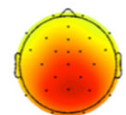

Emotiona

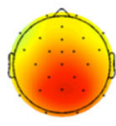

Neutral

Fig. 3 Pe data: Response-locked grand average waveforms at electrode $\mathrm{Pz}$ for correct and error trials with the emotional and with the neutral task set, for participants with low alexithymia (A) and participants with high alexithymia (B), as well as scalp topographies of the mean differences

difference between the Ne/ERN with the neutral and the emotional task sets) and alexithymia scores were calculated for each group separately. Correlations were calculated for each of the three TAS subscales: "Difficulties to Describe One's Own Feelings To Others" (DDF; e.g., "It is difficult for me to find the right words for my feelings"), "Difficulties to Identify Feelings and to Distinguish Them From the Somatic

\section{b High Alexithymia}

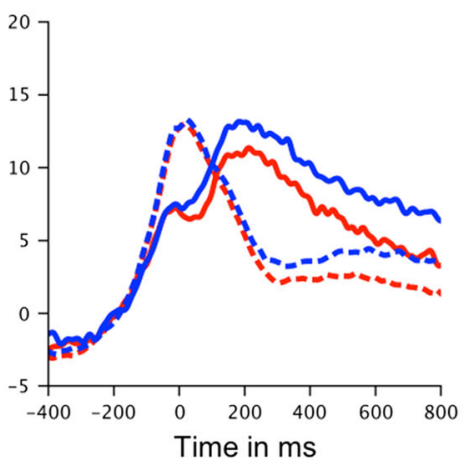

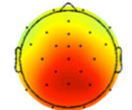

Emotional

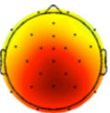

Neutral

\section{$8 \mu \mathrm{V}$ \\ $-8 \mu \mathrm{V}$}

between error and correct waveforms in a time window of 200 to $400 \mathrm{~ms}$ following the buttonpress $(\mathbf{C})$. $\mathrm{Pe}=$ error positivity, $0=$ time point of the buttonpress, $\mu \mathrm{V}=$ microvolts

Sensations That Accompany Emotional Arousal" (DIF; e.g., "I am often confused about what emotion I am feeling"), and "Externally Oriented Thinking" (EOT; e.g., "I prefer to analyze problems rather than just describe them"). These correlations are depicted in Fig. 4. In the LA group, no significant correlations were observed, all $p \mathrm{~s}>.067$. By contrast, in the HA group, a significant negative correlation between the $\mathrm{Ne} /$
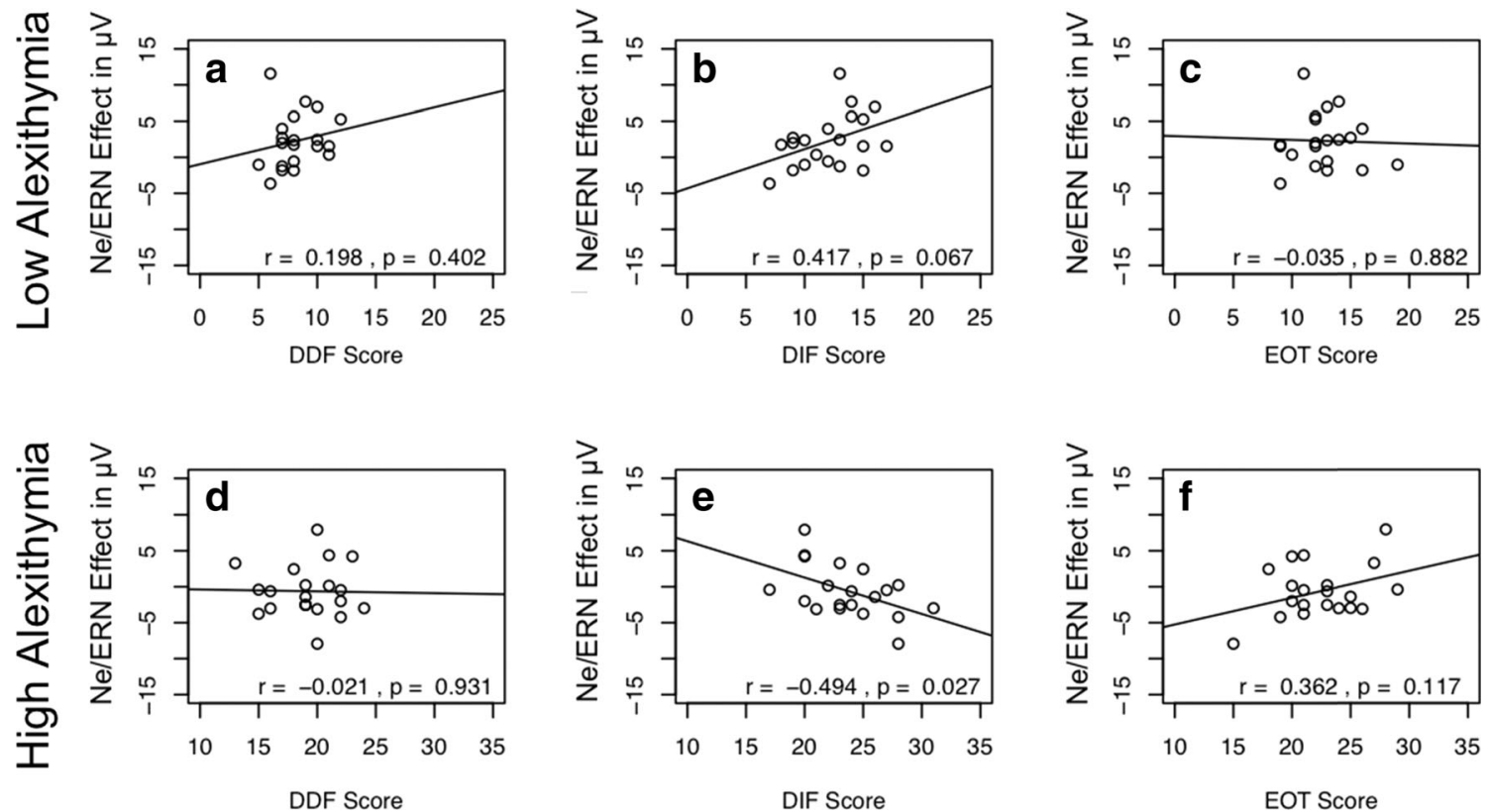

Fig. 4 Correlations between $\mathrm{Ne} / \mathrm{ERN}$ and difficulties with processing one's own affective states: Scatterplots and linear regression lines for the relationships between the effect of task set on the Ne/ERN (i.e., the difference in the Ne/ERN amplitudes between the emotional and neutral task sets) and individual scores on the subscales of the 20-item Toronto
Alexithymia Scale, for participants with low-alexithymia $(\mathbf{A}-\mathbf{C})$ and participants with high-alexithymia (D-F). DDF = difficulties describing feelings, DIF = difficulties identifying feelings, EOT $=$ external-oriented thinking, $\mathrm{Ne} / \mathrm{ERN}=$ error-related negativity, $r=$ Pearson correlation, $p=$ $p$ values, $\mu \mathrm{V}=$ microvolts 
ERN effect and the DIF subscale of the TAS was observed, $r=$ $-.494, p=.027$. Thus, HA participants who reported greater difficulty identifying their feelings showed a smaller increase in $\mathrm{Ne} / \mathrm{ERN}$ amplitude with the emotional task set, or even the reverse pattern of smaller Ne/ERN amplitudes with the emotional than with the neutral task set (see Fig. 4E). No other correlations were observed, all $p \mathrm{~s}>.117$.

To test, whether the correlations for the subscales differed between groups, we compared Fisher's z-transformed correlation coefficients between the LA and HA groups using twotailed $t$ tests. The only significant difference between groups emerged for the correlations between DIF and the effect of task set on the Ne/ERN effect, $p=.031$. No other differences were significant, $p \mathrm{~s}>.168$.

\section{Stimulus-locked ERP data}

N450 The waveforms seemed more positive for congruent than for incongruent stimuli (see Fig. 5). However, this effect seemed smaller for the HA group than for the LA group and for the emotional than for the neutral task set. A mixed-model repeated measures ANOVA with the between-subjects variable group (LA, HA) and the within-subjects variables task set (emotional, neutral) and congruency (congruent, incongruent) on the mean voltages in the N450 time window on correct trials confirmed these observations. The ANOVA revealed a main effect of congruency, $F(1,38)=75.9, p<.001, \eta_{\mathrm{p}}{ }^{2}=.666$, and interactions between task set and congruency, $F(1,38)=13.6$, $p<.001, \eta_{\mathrm{p}}{ }^{2}=.264$, and between group and congruency, $F(1$, $38)=5.42, p=.025, \eta_{\mathrm{p}}{ }^{2}=.125$. Waveforms were more positive for congruent than for incongruent trials (i.e., the N450 was present), with both the neutral (congruent, 14.2, $S E=$ 0.819 ; incongruent, $12.3, S E=0.828$ ) and the emotional (congruent, 14.3, $S E=0.841$; incongruent, 13.6, $S E=0.891$ ) task sets, $p \mathrm{~s}<.001, d \mathrm{~s}>0.128$. Thus, the interaction between task set and congruency denoted a larger N450 with the neutral task set. Similarly, the N450 was present both in the LA group (congruent, 14.0, $S E=0.670$; incongruent, 12.3, $S E=0.737$ ) and in the HA group (congruent, 14.5, $S E=0.941$; incongruent, $13.5, S E=0.968)$, $p \mathrm{~s}<.001, d \mathrm{~s}>0.159$. Thus, the interaction between group and congruency denoted that the N450 was smaller for the HA than for the LA group.

\section{Discussion}

The present study shows that error-monitoring processes are enhanced in emotional task contexts and that this effect depends on the ability to process internal affective states. Specifically, the Ne/ERN, an electrophysiological correlate
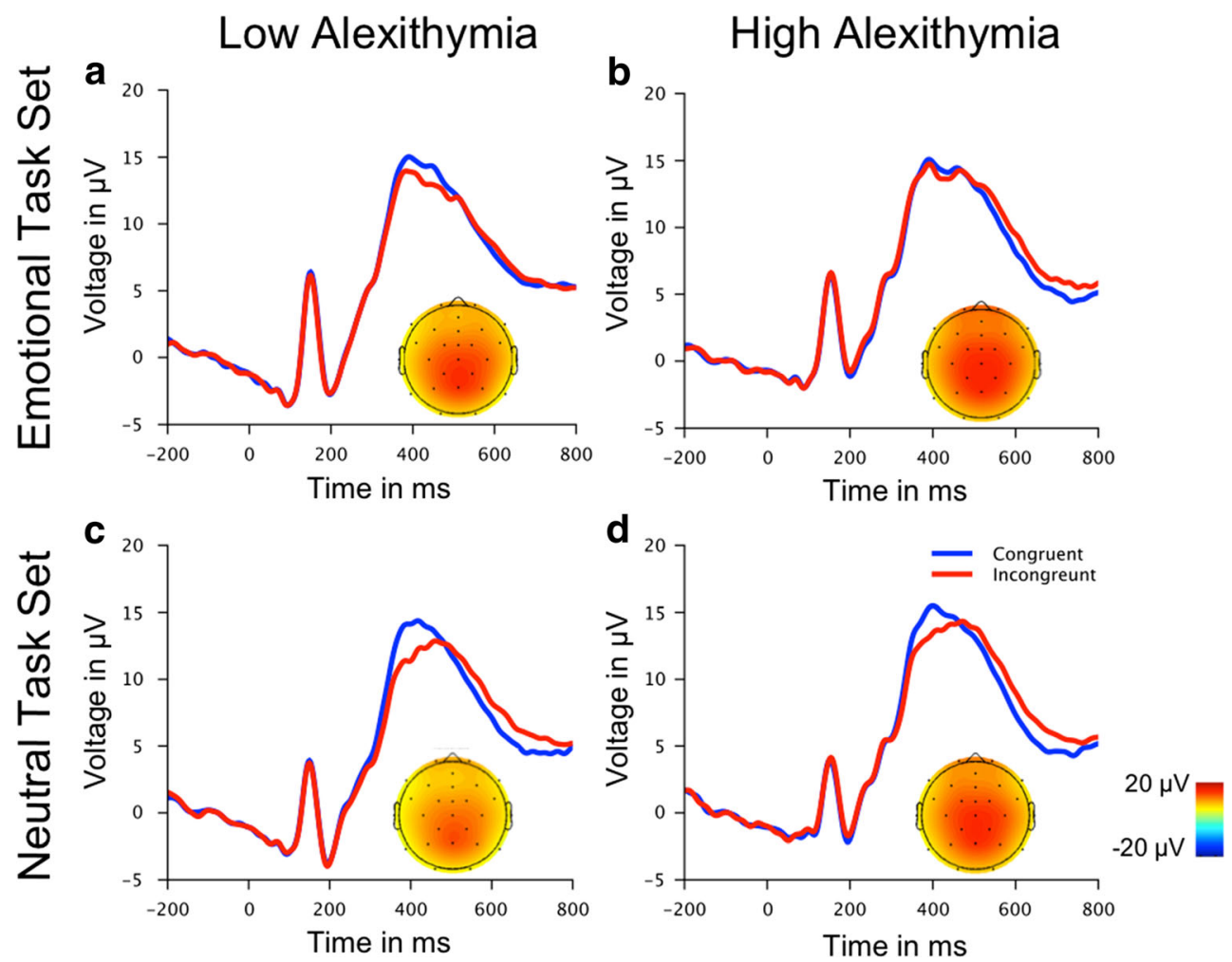

Fig. 5 N450 data: Stimulus-locked grand average waveforms at electrode $\mathrm{Pz}$ for congruent and incongruent correct trials with the emotional and the neutral task set, for participants with low alexithymia $(\mathbf{A}, \mathbf{C})$ and participants with high alexithymia $(\mathbf{B}, \mathbf{D})$, as well as scalp

topographies of the mean voltages in the time window of 350 to $450 \mathrm{~ms}$ following stimulus onset. $0=$ time point of stimulus onset, $\mu \mathrm{V}=$ microvolts 
of error monitoring, was larger in low-alexithymic participants, when they made errors with an emotional task set (i.e., when they classified emotional faces with respect to emotional expressions) than with a neutral task set (i.e., when they classified emotional faces with respect to gender). This indicates that the emotional task set elicits stronger errormonitoring activity. By contrast, high-alexithymic participants showed comparable Ne/ERNs with the emotional and the neutral task set. Moreover, the difference in the Ne/ERN between the task sets was negatively correlated with highalexithymic participants' difficulty in identifying their own feelings as measured by the Toronto Alexithymia Scale (Taylor et al., 2003). In other words, the more problems high-alexithymic participants had in identifying their own emotions, the less likely they were to show enhanced error monitoring with the emotional task set.

The result that participants with low alexithymia showed enhanced error-monitoring activity with the emotional task set is in accordance with previous studies, which found increased Ne/ERN amplitudes when the activity of the affective system was increased by presenting task-irrelevant affective information (e.g., Pfabigan et al., 2013; Wiswede, Münte, Goschke, \& Rüsseler, 2009). Note that the affective content of the task set enhanced exclusively the Ne/ERN on error trials, but did not affect the ERP in the Ne/ERN time window on correct trials. It has been suggested that in tasks in which participants must rely on feedback to evaluate their performance, a positive mediofrontal component following positive feedback rather than a negative component following negative feedback represents outcome monitoring processes (Holroyd, Pakzad-Vaezi, \& Krigolson, 2008). By contrast, our finding of exclusive modulation of the negativity on error trials shows that at least in the case of response monitoring, interactions between affective processes and monitoring processes are related more to the processing of negative than to the processing of positive outcomes.

Together, these findings support the notion that errormonitoring processes are closely linked to affective processes (Hajcak \& Foti, 2008; Luu et al., 2000). Extending previous literature, the result that no effect of the affective content of the task set on error monitoring was seen in participants with high alexithymia suggests that the relation between error monitoring and the affective pathways might depend on processing internal affective states. Thus, alexithymia might be a moderator of the relation between error monitoring and affective variables and could potentially explain the lack of consistency in the literature (e.g., Clayson, Clawson, \& Larson, 2012; Moser, Hajcak, \& Simons, 2005; Olvet \& Hajcak, 2012). Therefore, the present results suggest that future studies should include the assessment of alexithymia in participants when the task requires emotional processing or emotional reactivity.
The finding that difficulties in internal affective processing can eliminate the effect of the affective content of a task set on error monitoring suggests that the error-monitoring system uses information from the affective pathways to more efficiently signal error occurrence. On the one hand, the aMCC has close functional interconnections with core limbic structures implicated in affective processing (see Kober et al., 2008 , for a recent metaanalysis) and with motor centers associated with the expression of affect and the execution of goaldirected behavior. Therefore, it has been proposed that the aMCC integrates information about negative affect, pain and cognitive control in order to adaptively bias responding under uncertainty (Shackman et al., 2011). On the other hand, the aMCC is believed to signal the need to adjust performance following errors (Ridderinkhof et al., 2004; Ullsperger, Danielmeier, \& Jocham, 2014). Thus, a plausible scenario is that the aMCC integrates activity in affective pathways with error-monitoring processes such as monitoring violations of outcome predictions (Alexander \& Brown, 2011; Holroyd \& Coles, 2002) or postresponse conflict (Yeung et al., 2004). Such an integration could enable boosting the aMCC response to errors in the case of stronger responses in affective pathways. This would be adaptive, because it would be suited to more effectively signal errors that are particularly relevant for the organism. However, note that neither posterror slowing nor posterror increase of accuracy were modulated by the affective content of the task set or by alexithymia level. This suggests that these behavioral adjustments are not strongly dependent on activity in affective pathways.

The present results also extend our knowledge about alexithymia. First, like low-alexithymic participants, high-alexithymic participants did not make more errors with the emotional than with the neutral task set. This indicates that they did not have more difficulty in judging the emotional expression than in judging the gender of faces (see also Scarpazza et al., 2014). Second, high- and low-alexithymic participants also showed comparable congruency effects in behavior (i.e., differences in RTs and error rates between congruent and incongruent stimuli). The N450 as a correlate of conflict processing was even smaller in high- than in low-alexithymic participants, indicating less conflict at the neural level in alexithymia. Because the congruency effect is a measure of cognitive control, it can be concluded that high-alexithymic participants did not show a general decrement in cognitive control (but see De Galan et al., 2014). Interestingly, highalexithymic participants even showed a modulation of cognitive control by task set: Just like low-alexithymic participants, they showed smaller congruency effects with the emotional than with the neutral task set in both RT and error rate as well as in the conflict-related N450. Thus, the nature of the task set modulated cognitive control largely independently of alexithymia level. 
High-alexithymic participants demonstrated enhanced cognitive control as measured by the congruency effect with the emotional task set. By contrast, no enhancement of error monitoring as measured by the $\mathrm{Ne} / \mathrm{ERN}$ was observable with the emotional task set, and this seemed to be related to their difficulty in identifying feelings. This dissociation of cognitive control and error monitoring in high-alexithymic participants points to a selective impairment in alexithymic individuals of using the affective content of the task set to modulate error monitoring activity. Previous literature on the general population shows that error-monitoring processes and other measures of cognitive control are not only behaviorally, but also anatomically dissociable. A recent study using concurrent electroencephalogram and functional imaging found that $\operatorname{cog}$ nitive control as measured by the congruency effect is related to activity in the more dorsally located SMA, whereas error monitoring is related to activity in a larger portion extending from the SMA over the aMCC to more rostrally located rostral anterior cingulate cortex (Iannacone, Hauser, Staempfli, Walitza, \& Brem, 2015). The rostral anterior cingulate cortex shows altered activity in alexithymia (Bertoz et al., 2002; Frewen et al., 2008; Paradiso, Vaidyia, Cormick, Jones, \& Robinson, 2008; Sutherland, Carroll, Salmeron, Ross, \& Stein, 2013), and the present results indicate a reduced impact of emotions on error monitoring in alexithymia. Given the stronger involvement of the rostral anterior cingulate cortex in error monitoring than in interference control, our findings could suggest a role of this brain region in information transfer between the more dorsally located aspects of the errormonitoring system and the affective system.

Finally, we also investigated later parietal error-monitoring processes, indicating conscious error awareness (e.g., Steinhauser \& Yeung, 2010). Contrary to the difference found for the Ne/ERN, no effects of task set or alexithymia level were found for the Pe. Thus, the affective content of the task set and alexithymia level only affected rapid and automatic error monitoring at the time point of the erroneous response, but not later-emerging error awareness as measured by the Pe. Therefore, the present results imply that the processing of internal affective states is crucial for early automatic stages, but to a lesser degree for later deliberate stages, of error monitoring. Interestingly, from $200 \mathrm{~ms}$ following the response onward, high-alexithymic participants showed morenegative ERP waveforms with the emotional than with the neutral task set, irrespective of whether the response was correct or an error. No such effect was seen in low-alexithymic participants. It has been recently suggested that parietal positivity in this time range is a marker of decision confidence (Boldt \& Yeung, 2015). Thus, when adopting the emotional task set, highly alexithymic participants might have been less confident of their responses, irrespective of whether or not these were correct. However, because we collected no explicit measure of decision confidence, such as a confidence ratings, this idea remains speculative and should be tested in future studies.

In sum, the present study shows that error-monitoring activity as measured by the $\mathrm{Ne} / \mathrm{ERN}$ is stronger when adopting an emotional task set, and that this enhancement is related to the ability to process one's own affective states. This result is in line with the idea that the aMCC uses information from affective processing to boost early error-monitoring processes.

Author note This research was supported by a grant to Elisabetta Làdavas by the University of Bologna (FARB 2014, protocol number: RFBO120993). We thank Francesco di Gregorio and Daniele Migliorati for assistance in conducting the experiment and Marco Steinhauser and Neil Dundon for helpful comments on a previous version of this manuscript. The authors declare no competing financial interests.

\section{References}

Aarts, K., De Houwer, J., \& Pourtois, G. (2013). Erroneous and correct actions have a different affective valence: Evidence from ERPs. Emotion, 13, 960-973.

Alexander, W. H., \& Brown, J. W. (2011). Medial prefrontal cortex as an action-outcome predictor. Nature Neuroscience, 14, 1338-1344. doi:10.1038/nn.2921

Bell, A. J., \& Sejnowski, T. J. (1995). An information-maximization approach to blind separation and blind deconvolution. Neural Computation, 7, 1129-1159.

Bertoz, S., Artiges, E., Van de Moortele, P.-F., Poline, J.-B., Rouquette, S., Consoli, S. M., \& Martinot, J. L. (2002). Effect of impaired recognition and expression of emotions on frontocingulate cortices: An fMRI study of men with alexithymia. American Journal of Psychiatry, 159, 961-967.

Boldt, A., \& Yeung, N. (2015). Shared neural markers of decision confidence and error detection. Journal of Neuroscience, 38, 3478-3484.

Bonini, F., Burle, B., Liegéois-Chauvel, C., Régis, J., Chauvel, P., \& Vidal, F. (2014). Action monitoring and medial frontal cortex: Leading role of supplementary motor area. Science, 343, 888-891.

Botvinick, M. M., Braver, T. S., Barch, D. M., Carter, C. S., \& Cohen, J. D. (2001). Conflict monitoring and cognitive control. Psychological Review, 108, 624-652. doi:10.1037/0033-295X.108.3.624

Clayson, P. E., Clawson, A., \& Larson, M. J. (2012). The effects of induced state negative affect on performance monitoring processes. Social Cognitive and Affective Neuroscience, 7, 677-688.

De Galan, M., Sellaro, R., Colzato, L., \& Hommel, B. (2014). Conflict adaptation is predicted by the cognitive, but not the affective alexithymia dimension. Frontiers in Psychology, 22, 768. doi:10.3389/fpsyg.2014.00768

Debener, S., Ullsperger, M., Siegel, M., Fiehler, K., von Cramon, D. Y., \& Engel, A. K. (2005). Trial-by-trial coupling of concurrent electroencephalogram and functional magnetic imaging identifies the dynamics of performance monitoring. Journal of Neuroscience, 25, 11730-11737.

Delorme, A., \& Makeig, S. (2004). EEGLAB: An open source toolbox for analysis of single-trial EEG dynamics including independent component analysis. Journal of Neuroscience Methods, 134, 9-21. doi:10.1016/j.jneumeth.2003.10.009

Egner, T., Etkin, A., Gale, S., \& Hirsch, J. (2008). Dissociable neural systems resolve conflict from emotional versus nonemotional distracters. Cerebral Cortex, 18, 1475-1484. 
Etkin, A., Egner, T., \& Kalisch, R. (2011). Emotional processing in anterior cingulate and medial prefrontal cortex. Trends in Cognitive Sciences, 15, 85-93.

Falkenstein, M., Hohnsbein, J., Hoormann, J., \& Blanke, L. (1990). Effects of errors in choice reaction tasks on the ERP under focused and divided attention. In C. H. M. Brunia, A. W. K. Gaillard, \& A. Kok (Eds.), Psychophysiological brain research (Vol. 1, pp. 192195). Tilburg, The Netherlands: Tilburg University Press.

First, M. B., Spitzer, R. L., Gibbon, M., \& William, J. B. W. (2007). SCID-I: Structured Clinical Interview for DSM-IV Axis I Disorders (Versione italiana; F. Mazzi, P. Morosini, G. De Girolamo, M. Lussetti, \& G. P. Guaraldi, Eds.). Rome, Italy: Giunti O.S. (Original work published 1997)

Frewen, P. A., Lanius, R. A., Dozois, D. J. A., Neufeld, R. W. J., Pain, C., Hopper, J. W., ... Stevens, T. K. (2008). Clinical and neural correlates of alexithymia in posttraumatic stress disorder. Journal of Abnormal Psychology, 117, 171-181. doi:10.1037/0021-843 X.117.1.171

Gehring, W. J., Goss, B., Coles, M. G. H., Meyer, D. E., \& Donchin, E. (1993). A neural system for error detection and compensation. Psychological Science, 4, 385-390. doi:10.1111/j.1467-9280.1993. tb00586.x

Grandi, S., Sirri, L., Wise, T. N., Tossani, E., \& Fava, G. A. (2011). Kellner's emotional inhibition scale: A clinimetric approach to alexithymia research. Psychotherapy and Psychosomatics, 80, 335-344.

Hajcak, G., \& Foti, D. (2008). Errors are aversive: Defensive motivation and the error-related negativity. Psychological Science, 19, 103 108. doi:10.1111/j.1467-9280.2008.02053.x

Hajcak, G., McDonald, N., \& Simons, R. F. (2003). Anxiety and errorrelated brain activity. Biological Psychology, 64, 77-90.

Hajcak, G., McDonald, N., \& Simons, R. F. (2004). Error-related psychophysiology and negative affect. Brain and Cognition, 56, 189 197. doi:10.1016/j.bandc.2003.11.001

Hajcak, G., Moser, J. S., Yeung, N., \& Simons, R. F. (2005). On the ERN and the significance of errors. Psychophysiology, 42, 151-160.

Herbert, B. M., Herbert, C., \& Pollatos, O. (2011). On the relationship between interoceptive awareness and alexithymia: Is interoceptive awareness related to emotional awareness? Journal of Personality, 79, $1149-1175$.

Hintikka, J., Honkalampi, K., Lehtonen, J., \& Viinama, H. (2001). Are alexithymia and depression distinct or overlapping constructs? Comprehensive Psychiatry, 42, 234-239.

Holroyd, C. B., \& Coles, M. G. H. (2002). The neural basis of human error processing: Reinforcement learning, dopamine, and the errorrelated negativity. Psychological Review, 109, 679-709. doi:10.1037/0033-295X.109.4.679

Holroyd, C. B., Pakzad-Vaezi, K. L., \& Krigolson, O. E. (2008). The feedback correct-related positivity: Sensitivity of the event-related brain potential to unexpected positive feedback. Psychophysiology, 45, 688-697. doi:10.1111/j.1469-8986.2008.00668.x

Honkolampi, K., Hintikka, J., Tanskanen, A., Lehtonen, J., \& Viinamaki, H. (2000). Depression is strongly associated with alexithymia in the general population. Journal of Psychosomatic Research, 48, 99 104

Iannacone, R., Hauser, T. U., Staempfli, P., Walitza, S., \& Brem, S. (2015). Conflict monitoring and error processing: New insights from simultaneous EEG-fMRI. NeuroImage, 105, 395-407.

Jutten, C., \& Herault, J. (1991). Blind separation of sources, Part I: An adaptive algorithm based on neuromimetic architecture. Signal Processing, 24, 1-10.

Kaczkurkin, A. N. (2013). The effect of manipulating task difficulty on error-related negativity in individuals with obsessive-compulsive symptoms. Biological Psychology, 93, 122-131.

Kano, M., Fukudo, S., Gyoba, J., Kamachi, M., Tagawa, M., Mochizuki, H., ... Yanai, K. (2003). Specific brain processing of facial expressions in people with alexithymia: An H2 15O-PET study. Brain, 126, 1474-1484.

Karlsson, H., Näätänen, P., \& Stenman, H. (2008). Cortical activation in alexithymia as a response to emotional stimuli. British Journal of Psychiatry, 192, 32-38.

Kerns, J. G., Cohen, J. D., MacDonald, A. W. I., Cho, R. Y., Stenger, V. A., \& Carter, C. S. (2004). Anterior cingulate conflict monitoring and adjustments in control. Science, 303, 1023-1026. doi:10.1126/science.1089910

Kober, H., Feldman Barrett, L., Joseph, J., Bliss-Moreaur, E., Lindquist, K., \& Wager, T. D. (2008). Functional grouping and cortical-subcortical interactions in emotion: A meta-analysis of neuroimaging studies. NeuroImage, 42, 998-1031.

Kokkonen, P., Karvonen, J. T., Veijola, J., Läksy, K., Jokelainen, J., Järvelin, M. R., \& Joukamaa, M. (2001). Prevalence of sociodemographic correlates of alexithymia in a population sample of young adults. Comprehensive Psychiatry, 42, 471-476.

Larson, M. J., Clayson, P. E., \& Clawson, A. (2014). Making sense of all the conflict: A theoretical review and critique of conflict-related ERPs. International Journal of Psychophysiology, 93, 283-297.

Lundqvist, D., Flykt, A., \& Öhman, A. (1998). The Karolinska Directed Emotional Faces-KDEF (CD ROM). Stockholm, Sweden: Karolinska Institutet, Department of Clinical Neuroscience, Psychology section.

Luu, P., Collins, P., \& Tucker, D. M. (2000). Mood, personality, and selfmonitoring: Negative affect and emotionality in relation to frontal lobe mechanisms of error monitoring. Journal of Experimental Psychology: General, 129, 43-60. doi:10.1037/0096-3445.129.1.43

Maier, M. E., \& Steinhauser, M. (2013). Updating expected action outcome in the medial frontal cortex involves an evaluation of error type. Journal of Neuroscience, 33, 15705-15709.

Makeig, S., Bell, A. J., Jung, T.-P., \& Sejnowski, T. J. (1996). Independent component analysis of electroencephalographic data. In D. Touretzky, M. C. Mozer, \& M. Hasselmo (Eds.), Advances in neural information processing systems (Vol. 8, pp. 145-151). Cambridge, MA: MIT Press.

Mangelli, L., Semprini, F., Sirri, L., Fava, G. A., \& Sonino, N. (2006). Use of the Diagnostic Criteria for Psychosomatic Research (DPCR) in a community sample. Psychosomatics, 47, 143-146.

Moriguchi, Y., Decety, J., Ohnishi, T., Maeda, M., Mori, T., Nemoto, K., ... Komaki, G. (2007). Empathy and judging other's pain: An fMRI study of alexithymia. Cerebral Cortex, 17, 22232234. doi:10.1093/cercor/bhl130

Moser, J. S., Hajcak, G., \& Simons, R. F. (2005). The effects of fear on performance monitoring and attentional allocation. Psychophysiology, 42, 261-268.

Moser, J. S., Moran, T. P., Schroder, H. S., Donnellan, M. B., \& Yeung, N. (2013). On the relationship between anxiety and error monitoring: A meta-analysis and conceptual framework. Frontiers in Human Neuroscience, 7, 466. doi:10.3389/fnhum.2013.00466

Moser, J. S., Moran, T. P., Schroder, H. S., Donnellan, M. B., \& Yeung, N. (2014). The case for compensatory processes in the relationship between anxiety and error monitoring: A reply to Proudfit, Inzlicht, and Mennin. Frontiers in Human Neuroscience, 8, 64. doi:10.3389/fnhum.2014.00064

Olvet, D. M., \& Hajcak, G. (2012). The error-related negativity (ERN) relates to sadness following mood induction among individuals with high neuroticism. Social Cognitive and Affective Neuroscience, 7 , 289-295.

Overbeek, T. J. M., Nieuwenhuis, S., \& Ridderinkhof, K. R. (2005). Dissociable components of error processing: On the functional significance of the Pe vis-à-vis the ERN/Ne. Journal of Psychophysiology, 19, 319-329.

Paradiso, S., Vaidyia, J. G., Cormick, L. M., Jones, A., \& Robinson, R. G. (2008). Aging and alexithymia association with reduced right rostral cingulate. American Journal of Geriatric Psychiatry, 16, 760-769. 
Parker, J. D., Keefer, K. V., Taylor, G. J., \& Bagby, R. M. (2008). Latent structure of the alexithymia construct: A taxometric investigation. Psychological Assessment, 20, 385-396.

Pfabigan, D. M., Pintzinger, N. M., Siedek, D. R., Lamm, C., Derntl, B., \& Sailer, U. (2013). Feelings of helplessness increase ERN amplitudes in healthy individuals. Neuropsychologia, 51, 613-621.

Ridderinkhof, K. R., Ullsperger, M., Crone, E. A., \& Nieuwenhuis, S. (2004). The role of the medial frontal cortex in cognitive control. Science, 306, 443-447. doi:10.1126/science.1100301

Riesel, A., Weinberg, A., Endrass, T., Meyer, A., \& Hajcak, G. (2013). The ERN is the ERN is the ERN? Convergent validity of errorrelated brain activity across different tasks. Biological Psychology, 93, 377-385. doi:10.1016/j.biopsycho.2013.04.007

Riesel, A., Weinberg, A., Moran, T., \& Hajcak, G. (2013). Time course of error-potentiated startle and its relationship to error-related brain activity. Journal of Psychophysiology, 27, 51-59.

Salminen, J. K., Saarijarvi, S., Aarela, E., Toikka, T., \& Kauhanen, J. (1999). Prevalence of alexithymia and its association with sociodemographic variables in the general population of Finland. Journal of Psychosomatic Research, 46, 75-82.

Scarpazza, C., Di Pellegrino, G., \& Làdavas, E. (2014). Emotional modulation of touch in alexithymia. Emotion, 14, 602-610.

Scarpazza, C., Làdavas, E., \& Di Pellegrino, G. (2015). Dissociation between emotional remapping of fear and disgust in alexithymia. PLoS ONE, 10, e0140229. doi:10.1371/journal.pone.0140229

Schreiber, M., Endrass, T., Weigand, A., \& Kathmann, N. (2012). Age effects on adjustments of performance monitoring to task difficulty. Journal of Psychophysiology, 26, 145-153.

Shackman, A. J., Salomons, T. V., Slagter, H. A., Fox, A. S., Winter, J. J., \& Davidson, R. J. (2011). The integration of negative affect, pain and cognitive control in the cingulate cortex. Nature, 12, 154-167.

Simmons, J. P., Nelson, L. D., \& Simonsohn, U. (2011). False-positive psychology: Undisclosed flexibility in data collection and analysis allows presenting anything as significant. Psychological Science, 22, 1359-1366. doi:10.1177/0956797611417632

Steinhauser, M., \& Yeung, N. (2010). Decision processes in human performance monitoring. Journal of Neuroscience, 30, 15643-15653. doi:10.1523/JNEUROSCI.1899-10.2010

Sutherland, M. T., Carroll, A. J., Salmeron, B. J., Ross, T. J., \& Stein, E. A. (2013). Insula's functional connectivity with ventromedial prefrontal cortex mediates the impact of trait alexithymia on state tobacco craving. Psychopharmacology, 228, 143-155. doi:10.1007/s00213-013-3018-8

Taylor, G. J., Bagby, R. M., \& Parker, J. D. (1991). The alexithymia construct: A potential paradigm for psychosomatic medicine. Psychosomatics, 32, 153-164.

Taylor, G. J., Bagby, R. M., \& Parker, J. D. (1997). Disorders of affect regulation: Alexithymia in medical and psychiatric illness. Cambridge, UK: Cambridge University Press.

Taylor, G. J., Bagby, R. M., \& Parker, J. D. (2003). The 20-item Toronto Alexithymia Scale: IV. Reliability and factorial validity in different languages and cultures. Journal of Psychosomatic Research, 55, $277-283$.

Ullsperger, M., Danielmeier, C., \& Jocham, G. (2014). Neurophysiology of performance monitoring and adaptive behavior. Physiological Reviews, 94, 35-79. doi:10.1152/physrev.00041.2012

Viola, F. C., Thorne, J., Edmonds, B., Schneider, T., Eichele, T., \& Debener, S. (2009). Semi-automatic identification of independent components representing EEG artifact. Clinical Neurophysiology, $120,868-877$.

Weinberg, A., Olvet, D. M., \& Hajcak, G. (2010). Increased error-related brain activity in generalized anxiety disorder. Biological Psychology, 85, 472-480.

West, R., \& Alain, C. (2000). Effects of task context and fluctuations of attention on neural activity supporting performance of the Stroop task. Brain Research, 873, 102-111.

Winer, B. J., Brown, D. R., \& Michels, K. M. (1991). Statistical principles in experimental design. London, UK: McGraw-Hill.

Wiswede, D., Münte, T. F., Goschke, T., \& Rüsseler, J. (2009). Modulation of the error-related negativity by induction of shortterm negative affect. Neuropsychologia, 47, 83-90. doi:10.1016/j. neuropsychologia.2008.08.016

Wiswede, D., Münte, T. F., \& Rüsseler, J. (2009). Negative affect induced by derogatory verbal feedback modulates the neural signature of error detection. Social Cognitive and Affective Neuroscience, 4, 227-237. doi:10.1093/scan/nsp015

Yeung, N., Botvinick, M. M., \& Cohen, J. D. (2004). The neural basis of error detection: Conflict monitoring and the error-related negativity. Psychological Review, 111, 931-959. doi:10.1037/0033-295 X.111.4.931 